\title{
Desenvolvimento Econômico na Região da Riba a partir da Relação Crescimento-Desigualdade sob Hipótese(s) da(s) Curva(s) de Kuznets
}

\author{
Rhayza Alves Figueiredo de Carvalho'; Abner Vilhena de Carvalho '; Jarsen Luis Castro \\ Guimarães ${ }^{l}$ \\ \} \text { carvalho.rhayza@gmail.com }
}

1. Universidade Federal do Oeste do Pará (UFOPA), Pará, Brasil.

\section{Histórico do Artigo:}

Recebido em: 09 de maio de 2020 Aceito em: 03 de agosto de $2020 \quad$ Publicado em: 31 de dezembro de 2020

Resumo: 0 presente ensaio teve como objetivo analisar a possível relação entre o crescimento econômico e a desigualdade na Região de Integração do Baixo Amazonas (RIBA), no período de 2000 a 2010. Por meio de investigação da(s) hipótese(s) da curva de Kuznets e, a partir da construção de um modelo econométrico com dados em painel curto, usou-se como proxies da desigualdade e do crescimento econômico os seguinte indicadores: 0 índice de Gini (variável dependente) e a renda per capita (variável independente), sendo esta última utilizado sob a forma linear, cúbica e quadrática, a fim de testar os possíveis resultados das curva no formato de U-invertido e $\mathrm{N}$, de modo a contribuir para a discussão no campo da economia do desenvolvimento na região. Os resultados do modelo apontaram para evidência da hipótese do U-invertido da curva de Kuznets presente no primeiro estágio do progresso econômico na região, e o crescimento econômico atuando como pacificador momentâneo junto à desigualdade de renda na RIBA.

Palavras-chave: Crescimento, Desigualdade, Curva de Kuznets, U-invertido e N, RIBA.

\section{Economic Development in the Riba Region from the Growth-Inequality Relationship under the Kuznets Curve(S) Hypothesis}

Abstract: The present essay aimed to analyze the possible relationship between economic growth and inequality in the Integration Region of the Lower Amazon (RIBA), in the period from 2000 to 2010. Through the investigation of the hypothesis (s) of the Kuznets and, based on the construction of an econometric model with data in a short panel, the following indicators were used as proxies for inequality and economic growth: the Gini index (dependent variable) and per capita income (independent variable), the latter being used in a linear, cubic and quadratic form, in order to test the possible results of the curves in the shape of inverted $\mathrm{U}$ and $\mathrm{N}$, in order to contribute to the discussion in the field of development economics in the region. The results of the model pointed to evidence of the Kuznets curve inverted $U$ hypothesis present in the first stage of economic progress in the region, and economic growth acting as a momentary peacemaker with income inequality in RIBA.

Keywords: Growth, Inequality, Kuznets Curve, U-inverted and N, RIBA. 

sob Hipótese(s) da(s) Curva(s) de Kuznets

\section{Desarrollo Económico en la Región de Riba desde la Relación de Desigualdad de Crecimiento bajo la Hipótesis de la Curva(S) De Kuznets}

Resumen: El presente ensayo tuvo como objetivo analizar la posible relación entre el crecimiento económico y la desigualdad en la Región de Integración de la Baja Amazonía (RIBA), en el período de 2000 a 2010. A través de la investigación de la (s) hipótesis (s) de la Kuznets y, en base a la construcción de un modelo econométrico con datos en un panel corto, los siguientes indicadores se utilizaron como indicadores de desigualdad y crecimiento económico: el índice de Gini (variable dependiente) y el ingreso per cápita (variable independiente), esta última se usa en forma lineal, cúbica y cuadrática, para probar los posibles resultados de las curvas en forma de $\mathrm{U}$ y $\mathrm{N}$ invertidos, para contribuir a la discusión en el campo de la economía del desarrollo en la región. Los resultados del modelo apuntaban a la evidencia de la hipótesis de la U invertida de la curva de Kuznets presente en la primera etapa del progreso económico en la región, y el crecimiento económico actuando como un pacificador momentáneo con desigualdad de ingresos en RIBA.

Palabras clave: Crecimiento, Desigualdad, Curva de Kuznets, U invertida y N, RIBA.

\section{INTRODUÇ̃̃o}

0 desenvolvimento gera melhorias socioeconômicas que desencadeiam aumento nos padrões de vida também nos níveis de bem estar da população. Desse modo, uma das preocupações mais relevantes das autoridades governamentais deve ser efetivar ações de políticas que resultem nos referidos benefícios sociais. Nesse sentido, uma das vertentes analíticas, sugere que o crescimento econômico pode contribuir na mobilidade entre os estratos da população com menores níveis de renda e na interrupção de fenômenos como a pobreza e a distribuição de renda.

Souza (1997, p. 28) salientava que o "desenvolvimento econômico era definido pelo aumento contínuo dos níveis de vida incluindo maior consumo de produtos e de serviços básicos para o conjunto da população”. Nesse sentido, estudar a tríade do desenvolvimento - relação entre os fenômenos do crescimento econômico, desigualdade e pobreza, deve-se principalmente verificar como essas variáveis (e suas proxies) se relacionam e geram impactos entre si, tanto positivos quanto negativos, possibilitando compreender a dinâmica social e investigação de novos rumos a seguir ruma às mudanças no cenário atual do Brasil e regiões.

A íntima relação entre crescimento, desigualdade e pobreza pode ser comprovada pela própria dinâmica que o sistema econômico capitalista foi desencadeado, seguido de eficiência na produção de bens, entretanto, ocasionando males sociais e ambientais, com rápido crescimento impulsionado por empresas modernas que tendem a ser concentradoras e excludentes. 
0 conceito de desenvolvimento emerge como forma de minimizar desigualdades que foram acumuladas ao longo do tempo, na qual afirma Sachs (2008, p. 13) ser possível criar “[...]uma conexão capaz de preencher 0 abismo civilizatório entre as antigas nações metropolitanas e a sua antiga periferia colonial, entre ricas modernidades e a maioria ainda atrasada e exausta dos trabalhadores pobres”. Mudanças de padrões podem gerar benefícios sem precisamente requerer crescimento econômico, provocando alterações que diminuam os desvios, orientando na direção de atividades produtivas que podem induzir ao investimento eficiente, acumulação de qualificações, transferência de tecnologia, e a própria infraestrutura dentro de uma localidade podem levar a mudanças do nível de renda (JONES, 2000).

Inúmeros são os adjetivos relacionados a ideia de desenvolvimento, devido sua complexidade e multidimensionalidade. Esse conceito exige uma tendência de crescimento econômico, impactos sociais e ambientais positivos, constituindo um dos mais variados padrões de crescimento econômico observado ao longo do tempo. Portanto, o crescimento, não se converte em desenvolvimento caso não amplie o nível de emprego, não reduza a pobreza e não atenua as desigualdades (SACHS, 2008).

Por conseguinte, a maioria da população que vive em situação de pobreza não usufrui do acesso à melhor educação, não compreendendo na totalidade os direitos humanos (civis, cívicos e políticos) e logo, sendo impossibilitada de obter um trabalho decente, e da mesma maneira, um nível digno de saúde e moradia dignas, desse modo, a porção é excluída do processo de desenvolvimento, que requer a redistribuição de uma parcela do PIB por meio dos entes públicos, caracterizado assim, um crescimento excludente e concentrador (VEIGA, 2010).

Souza (1997, p. 28) enfatiza que "Medidas destinadas a atacar diretamente a pobreza podem ser indispensáveis quando a renda for muito concentrada e as populações carentes muito numerosas”. Portanto, um maior nível de renda por vezes, pode não se traduzir necessariamente em melhores índices de desenvolvimento.

Existem algumas formas de mensurar essa relação tríade (crescimento econômicodesigualdade-pobreza), principalmente a fim de investigar a contribuição e o direcionamento das políticas públicas adotadas com foco na promoção do desenvolvimento econômico. A relação entre crescimento e redução da pobreza pode ser medida pela elasticidade-renda ou elasticidade-crescimento, de modo que, se a elasticidade é elevada, políticas públicas de combate à pobreza baseadas no crescimento econômico são mais eficientes. Caso contrário, se a elasticidade é baixa, estratégias de redução da pobreza deveriam envolver uma combinação 
Desenvolvimento Econômico na Região da Riba a partir da Relação Crescimento-Desigualdade sob Hipótese(s) da(s) Curva(s) de Kuznets

de crescimento econômico com algum tipo de redistribuição de renda (SILVA; OTTONELLI; MARIN, 2013).

Na literatura especializada, durante muito tempo os fenômenos do crescimento e do desenvolvimento econômico foram utilizados como sinônimos. Contudo, na atualidade, parece ser mais compreensível que se trata de visões distintas, porém não deixando de ser complementares.

No contexto dessas discussões entre crescimento econômico e os impactos sobre os níveis de desigualdade, este trabalho visa verificar a existência de evidência da curva de Kuznets Tradicional (CKT). Dessa forma, foi construído um modelo econométrico com dados em painel curto com o objetivo de investigar a relação entre tais variáveis, utilizando como proxies o PIB e nível de desigualdade de renda nos municípios que integram a Região de Integração do Baixo Amazonas (RIBA), com microdados dos Censos Demográficos (IBGE), durante o período de 1991, 2000 e 2010.

0 objetivo foi verificar se 0 crescimento econômico da região impacta na diminuição da desigualdade, ou seja, se o crescimento econômico tende a aliviar problemas de desigualdade, tendo como principal finalidade responder a seguinte questão: A hipótese de Kuznets é (são) válida(s) para a RIBA no período analisado?

\section{REFRENCIAL TEÓRIC0 E REVISÃ0 DA LITERATURA}

\section{Desenvolvimento econômico e a hipótese do "U invertido" da Teoria de Kuznets}

A realidade socioeconômica do Brasil é marcada por graves problemas sociais, como altas concentrações de renda, pobreza, desigualdade entre outros impasses sociais, que têm sido foco de várias ações governamentais e políticas públicas, com o intuito de minimizar os respectivos efeitos maléficos sobre a qualidade de vida da população. A importância desse estudo é comprovada pelos diferentes contextos, que são alvo de estudos empíricos e, que buscam ao longo da literatura explicar essa relação entre desigualdade e crescimento econômico para diferentes realidades sociais.

Dessa forma, algumas ações governamentais e certos instrumentos são extremamente relevantes para tentar minimizar problemas sociais que se revelam duradouros, como a extrema desigualdade na distribuição de renda. Portanto, a corroboração da hipótese do "U" invertido para determinadas localidades pode ser considerada um fator de demarcação, ou seja, um instrumento que proporciona um limite no direcionamento de novas políticas públicas, pois a grande heterogeneidade entre as localidades exige a necessidade da existência de programas 
direcionados de modo particular a cada perfil socioeconômico, além de outras ações, como por exemplo, o abandono de políticas de cima para baixo, denominadas de Top-Down (LINHARES et al., 2012).

A análise da trajetória de variáveis socioeconômicas e do comportamento entre elas podem revelar causalidades essenciais, proporcionando entendimento sobre suas dinâmicas que afetam diretamente as condições de vida da população.

Para fins analíticos, partindo de uma dada distribuição inicial de renda e da desigualdade dessa distribuição medida pelo índice de Gini, pode-se, naquele momento, verificar como esta evolui a partir de um determinado processo de crescimento econômico sustentado. Logo, o ponto de partida analítico pode ser o efeito de como essa distribuição inicial influenciou o processo de crescimento, ou pode-se verificar como o crescimento impactou a distribuição e, desta forma, a desigualdade (ARRAES; DINIZ; DINIZ, 2006).

Silva (2010) salienta que políticas sociais com maiores resultados devem estar associadas a políticas macroeconômicas, para que possibilite um crescimento econômico sustentado, geração de emprego, elevação do nível de renda proveniente do trabalho e, sobretudo, de redistribuição de renda.

Nesse sentido, o estudo realizado por Kuznets (1955) se tornou referência teórica acerca da relação entre crescimento econômico e desigualdade de renda, no qual comparando tais fenômenos sugeriu uma relação não linear, por meio de um estudo empírico com series de tempo para países desenvolvidos e subdesenvolvidos, indicando que no curto prazo a desigualdade de renda tende a aumentar nos primeiros estágios de crescimento, até atingir um certo ponto, correspondendo a um determinado nível de renda per capita, o ponto máximo, a partir do qual, ao longo do tempo ocorreria um movimento descendente no sentido em que a economia iria se desenvolvendo, gerando uma redução na desigualdade. Portanto, a proposta era observar a questão da desigualdade de renda nos diferentes estágios de crescimento econômico (KUZNETS, 1955; SALVAT0 et al., 2006; LINHARES et al., 2012).

No estágio inicial a relação tende a ser uma curva positivamente inclinada, porém, em um dado ponto essa curva tenderia a se tornar negativa, sendo denominada na literatura como hipótese do "U" invertido ou "Curva de Kuznets". 0 estudo foi o ponto de partida para vários trabalhos, que passaram a verificar padrões de crescimento, em distintas localidades e temporalidades, utilizando diferentes metodologias, e permitindo assim, verificar a dinâmica de variáveis essenciais que compõem o processo de desenvolvimento de uma sociedade (KUZNETS, 1955). 
Desenvolvimento Econômico na Região da Riba a partir da Relação Crescimento-Desigualdade sob Hipótese(s) da(s) Curva(s) de Kuznets

Essa relação configurada em U invertido com 5\% de informações empíricas e 95\% de especulação. Além disso, comparou-se a estrutura de renda entre os séculos XIX e XX das sociedades consideradas desenvolvidas, tais como: Estados Unidos, Grã-Bretanha, Prússia e Saxônia (Alemanha). Ao comparar com outras nações, subdesenvolvidas, Kuznets percebeu a existência de uma maior desigualdade nestas últimas nações do que nas desenvolvidas, levantando 0 questionamento sobre a existência de um mesmo padrão para sociedades subdesenvolvidas, e ressaltando também a limitação de dados para testar a realidade observada nestes países, que apesar de especulativo considerou a resposta afirmativa em seu trabalho (KUZNETS, 1955).

Ao longo do processo de crescimento econômico podem surgir melhores oportunidades e benefícios, como maiores acessos aos sistemas de saúde e educação para as sociedades democráticas, bem como maiores consciências de direitos e deveres políticos. Proporcionando acréscimo na produtividade e diminuindo as desigualdades, de modo a atenuar os resultados da rápida industrialização e urbanização (SALVAT0 et al., 2006 apud KUZNETS, 1955).

A explicação para tal fato decorreria da transferência da população do setor agrícola, para o setor urbano, mais moderno e industrializado, essa troca entre setores promoveria um aumento da desigualdade, pois a produtividade per capita nas atividades industriais cresce mais rápido do que na agricultura, gerando uma elevação na desigualdade até que o produto per capita atingisse um determinado ponto, em que ocorreria uma melhor distribuição desses rendimentos e a desigualdade permaneceria estável, em seguida, ocorreria uma tendência de diminuição contínua à medida que a renda per capita permanecesse evoluindo de forma crescente. Além de argumentos como imperfeição no mercado de capitais, até a inclusão da tecnologia como determinante da desigualdade de rendimentos (SALVATO et al., 2006).

Alguns autores acreditam que o interesse em definir a relação entre desigualdade de renda e desenvolvimento econômico são fundamentados de acordo com a forma de abordagem, tais como: modelos de economia dual proposto por Kuznets, modelos new-growth ligados, com a teoria de mercados de capitais imperfeitos e com a importância do investimento em capital humano, e por fim modelos de escolha pública, no qual envolvem a interação entre o mecanismo econômico e o político (DEUTSCH; SILBER, 2000; SALVAT0 et al., 2006).

Assim "a ideia básica era que a distribuição de renda tem que ser pensada como uma combinação da distribuição de renda da população rural e urbana, onde esta última, apesar de deter uma renda média mais alta, apresenta maior concentração do que a primeira”. Além do mais, "quando a renda cresce o diferencial de renda per capita entre as áreas rural e urbana 
persistiria, ou mesmo alargaria, uma vez que o crescimento da produtividade na área urbana seria maior que na área rural” (ARRAES; DINIZ; DINIZ, 2006, p. 527).

Deutsch e Silber (2000) acreditam que o interesse pelas pesquisas entre a ligação da desigualdade de renda e desenvolvimento econômico se justifiquem por três fatores como: o surgimento das teorias new-growth, a disponibilidade de dados mais sofisticados e a aplicação da teoria da escolha pública em novas áreas.

Ressalta-se que nacionalmente os principais estudos que testam empiricamente a hipótese da curva de Kuznets utilizam dados de cross-section, sendo considerada uma metodologia tradicional ao tema, além dos dados serem baseados no censo do país (LINHARES et al., 2012).

Apesar da discordância quanto à limitação do modelo econométrico para dados em cross-section, alguns autores acreditam que tal forma desconsideraria as diferenças históricas particulares de cada localidade nas trajetórias de evolução da renda e da desigualdade, tendenciado assim, a obtenção de resultados errôneos para a curva estimada do U invertido ou normal (SALVAT0 et al., 2006).

\section{Evidência da hipótese do "U invertido” de Kuznets - revisão empírica}

Dentre os trabalhos efetuados para o Brasil, que buscaram evidências para a validação, ou não, da hipótese de Kuznets, tem-se o estudo de Salvato et al. (2006) que analisou a relação entre a desigualdade de renda e o desenvolvimento econômico para os municípios de Minas Gerais nos anos 1991/2000, utilizando como proxies a renda municipal per capita mensal e os índices de Theil - L e Gini, por meio de metodologias de cross-section e dados em painel, para a desigualdade em função da renda na forma quadrática. Seus resultados mostraram que no ano de 1991 existiu evidências do "U invertido" nos modelos cross-section para ambos indicadores, contrários aos obtidos em 2000, que seguiram o padrão de um U-normal. Nos dados em painel, encontraram o padrão do U-invertido para os municípios no período 1991/2000, tanto para o Índice L de Theil quanto para o Índice de Gini.

Santos et al. (2011) investigaram se a hipótese do "U-invertido" se aplica para o Brasil, no período de 1976-2007, utilizando com proxies a desigualdade e o crescimento econômico: 0 índice de Gini e de Theil - T e a renda domiciliar per capita média, além de uma modelagem em series temporais na forma quadrática e auto regressivo de primeira ordem. Evidenciou-se a existência de uma curva de Kuznets com U invertido, para estimações tanto para o índice de Gini como o de Theil. 
Desenvolvimento Econômico na Região da Riba a partir da Relação Crescimento-Desigualdade sob Hipótese(s) da(s) Curva(s) de Kuznets

Santos, Cunha e Gadelha (2017) buscaram verificar se a relação entre desenvolvimento econômico e distribuição de renda em 26 estados brasileiros no período de 1992-2010 seguiu o formato da curva de Kuznets, por meio de dados em painel, sob a forma funcional da renda per capita linear, quadrática e Log-Log. Os resultados encontrados no estudo mostram que os dados em painel estático confirmaram a hipótese de Kuznets, de modo que apesar da grande diferença entre os estados, em um primeiro momento o nível de atividade econômica afeta positivamente a desigualdade e, posteriormente, a partir de um estágio de maior renda per capita, a atividade econômica passa a afetar negativamente a desigualdade.

Barbosa et al. (2017) analisaram empiricamente a relação de U invertido de Kuznets, utilizando dados em painel, censitários de 1991, 2000 e 2010 para o Brasil analisando os estados, sob a forma funcional quadrática e cúbica com modelo Log-Log. Utilizou como proxies a renda domiciliar per capita, o índice de Gini, além da inclusão de variáveis como a população urbana e a população rural além da expectativa de anos de estudo. Um dos principais resultados é a existência da curva em formato de U invertido, somente no termo quadrático, no qual ocorreu aumento na desigualdade de renda até os anos 2000, declinando no final da década, já em 2010.

Contudo, resultados contrários à corroboração da hipótese do U-invertido de Kuznets foram encontrados nos trabalhos de Taques e Mazzutti (2009) em que as evidências da relação entre desigualdade de renda e crescimento econômico (proxies - renda per capita e os índices de Theil - L e Gini) para os Estados do Brasil entre 1995 e 2005, em sua forma funcional quadrática e modelo em Log-log e semi-log, onde não foi confirmada a hipótese de Kuznets, existindo um padrão de U normal estatisticamente significante nos modelos que consideram o índice de Gini, enquanto que para o índice L de Theil os resultados da maioria dos modelos apresentaram o padrão de U invertido, porém, com coeficientes não significantes.

Outro trabalho de Taques (2010) que testou a hipótese de Kuznets para unidades federativas brasileiras no período entre 1995 e 2008, obtendo os mesmos resultados do estudo anterior por meio dos mesmos procedimentos econométricos com mesmas proxies, evidenciando pouco suporte para corroboração da hipótese.

Linhares et al. (2012) investigaram a hipótese de Kuznets para 21 estados do Brasil, com dados em painel, no período de 1986 a 2005, utilizando as proxies: índice de Gini, renda per capita, além de incluírem outras como: razão entre a soma das exportações com importações em relação ao PIB, o consumo de energia elétrica industrial e um referente a média de anos de estudo dos estados, por meio de uma forma quadrática e outra cúbica para renda per capita. $0 \mathrm{~s}$ resultados mostraram a existência de três regimes de nível de renda per capita: o primeiro 
inferior a R\$ 258,48 mensais; um segundo (intermediário) iguais ou maiores do que R\$ 258,48 e menores do que $\mathrm{R} \$ 395,62$ mensais, e por fim; o terceiro com superiores a $\mathrm{R} \$ 395,62$ mensais, observando a relação de U invertido de Kuznets no segundo e terceiro regime.

Tabosa, Filho e Gomide (2016) averiguaram a existência da hipótese da curva de Kuznets para o Brasil no período de 1981 a 2009, com dados em painel, e proxies de crescimento econômico e desigualdade de renda as respectivas renda domiciliar per capita média e os indicadores de Gini e Theil, por meio das formas funcionais linear, quadrática e cúbica para a renda. De forma geral, os resultados mostraram a falta de evidências empíricas para a confirmação da hipótese de Kuznets.

0 estudo de List e Gallet (1999) foi além das especificações tradicionais, e realizou análises econométricas mais sofisticada, como um polinômio de terceiro grau para a renda per capita, acrescentando uma variável de tendência, com intuito de examinar os acontecimentos posteriores a curva de Kuznets, além de verificar um possível retorno no aumento da desigualdade. 0s resultados obtidos sugeriram que a partir de um determinado nível de renda per capita, a desigualdade volta a subir.

\section{METODOLOGIA}

\section{Área de abrangência}

A(s) hipótese(s) da(s) curva(s) de Kuznets foi testada para a Região de Integração do Baixo Amazonas, composta por treze municípios do estado do Pará, que são: Alenquer, Almeirim, Belterra, Curuá, Faro, Juruti, Mojuí dos Campos, Monte Alegre, Óbidos, Oriximiná, Prainha, Santarém e Terra Santa (FAPESPA, 2017).

Vale ressaltar que o município de Mojuí dos Campos foi integrado RIBA no ano de 2012, e por isto não foi incorporado nas análises do estudo.

\section{Origem dos dados - periodicidade e as variáveis (proxies) do modelo}

Considerou-se o PIB per capita (variável explicativa) e o índice de Gini (variável dependente), como proxies do crescimento econômico e da desigualdade de renda, encontrados no Atlas de Desenvolvimento Humano (disponível em http://atlasbrasil.org.br/2013/) elaborado pela Fundação João Pinheiro e referente aos municípios da RIBA, proveniente dos microdados dos Censos Demográficos dos anos de 1991, 2000 e 2010 (três período de 10 anos), visando à 
Desenvolvimento Econômico na Região da Riba a partir da Relação Crescimento-Desigualdade sob Hipótese(s) da(s) Curva(s) de Kuznets

observação da influência dos diversos contextos sobre as variáveis consideradas no modelo de análise.

\section{Metodologia de análise e descrição dos modelos dos utilizados}

A proposta de Kuznets (1955) inspirou muitos pesquisadores em estudar as relações associativa entre o nível de crescimento econômico, desigualdade de renda e outras variáveis, sob a hipótese da forma de "U invertido" das CKT e outras curvas. Contudo, conforme afirma Ahluwalia (1976) citado por Tabosa, Filho, Gomide (2016, p. 249) "tais relações são mais bem descritas como 'fatos estilizados', que podem ser observados, mas, no entanto, necessitam de formulações teóricas para explicá-las”.

Diversas abordagens econométricas foram empregadas a fim de se testar a hipótese do “U invertido”, e percebe-se que, em geral, não há um modelo teórico estabelecido que sugira um conjunto de regressores que devam ser incluídos na estimação do modelo. Dentre os estudos, averiguou-se que a forma funcional mais usual é o polinômio de $2^{\circ}$ grau, embora estudos atuais se utilizem da forma de $3^{\circ}$ grau.

Os modelos polinomiais, representados graficamente por uma curva na forma de "U" parábola, mostra que a relação entre $X$ e $Y$ não é linear. Dito de outra maneira, este tipo de modelo captaria, primeiro, a queda e, depois o aumento nos pontos de dispersão das variáveis relacionadas (GUJARATI, 2006; GUJARATI; PORTER, 2011). Matematicamente, a parábola é representada pela equação (1), conhecida como função quadrática ou, de modo mais geral, polinômio de segundo grau na variável $X$ (GUJARATI, 2006; GUJARATI; P0RTER, 2011). A versão estocástica, daquele polinômio de segundo grau, denominada regressão polinomial de segundo grau, é representada sob a forma equação (2).

$Y=\beta_{0}+\beta_{1} X+\beta_{2} X^{2}$

$Y_{i t}=\beta_{0}+\beta_{1} X_{i t}+\beta_{2} X_{i t}^{2}+u_{t}$

Na equação polinomial, Gujarati (2006, p. 182) “o expoente mais alto de $X$ representa 0 grau do polinômio”. Se acrescentássemos, por exemplo, $X^{3}$ à função da equação (1), teríamos um polinômio de terceiro grau e, $X_{i t}^{3}$ à função da equação (2), teríamos dessa maneira, uma regressão polinomial de terceiro grau. Assim, a forma geral da regressão polinomial de k-ésimo grau, conforme Gujarati (2006, p. 182) é:

$Y_{i t}=\beta_{0}+\beta_{1} X_{i t}+\beta_{2} X_{i t}^{2}+\cdots+\beta_{k} X_{i t}^{k}+u_{t}$ 
Destaca-se que, nos diversos tipos de regressão polinomial só há uma variável explanatório do lado direito, mas ela aparece elevada a várias potencias, o que, necessariamente o torna um modelo de regressão múltipla. Nesse contexto, ressalta-se também, que não podemos esquecer que termos como $X_{2}, X_{3}, X_{4}$ e etc. são todos eles funções não lineares de $X$ e, portanto, rigorosamente não desrespeitam a premissa da ausência da multicolinearidade. 0u seja, já que o polinômio de $k$-ésimo grau é linear nos parâmetros, os $\beta$ dos modelos de regressão polinomial podem ser estimados pelo método de mínimos quadrados ordinários (MMQ0) ou de método da máxima verossimilhança (MMV) (GUJARATI, 2006).

A especificação geral do modelo foi estimada como:

Gini $=\beta_{0}+\beta_{1}$ Rendapc $_{i t}+\beta_{2}$ Rendap $_{i t}^{2}+\beta_{3}$ Rendapc $_{i t}^{3}+\varepsilon_{i t}$ Onde:

Gini: representa o indicador de desigualdade - Índice de Gini;

$\beta_{0}, \beta_{1}, \beta_{2}, \beta_{3}$ : são os parâmetros das rendas nos modelos (vetores $k_{x} l, \mathrm{com} \mathrm{i}=1,2,3$ ).

$\beta_{1} \operatorname{Rendapc}_{i t}, \beta_{2} \operatorname{Rendapc}_{i t}^{2}, \beta_{3} \operatorname{Rendapc}_{i t}^{3}$ : representa o PIB per capita nas formas linear, quadrática e cúbica, respectivamente. $\varepsilon_{i t}$ : é o termo do erro (resíduos) do modelo.

0 índice de Gini (que mede a desigualdade de renda) é a variável de interesse, $Y$, e a renda média domiciliar per capita (nas formas linear, quadrática e cúbica), X’'s, as explicativas. № quadro (1), está a descrição das variáveis (proxies) do modelo.

Quadro 1. Descrição das variáveis utilizadas nos Modelos de Kuznets

\begin{tabular}{|c|c|c|c|c|c|}
\hline & Variável (proxy) & Descrição & Unidade & $\begin{array}{c}\text { Sinal } \\
\text { esperado }\end{array}$ & Fonte \\
\hline $\begin{array}{c}\text { Variável } \\
\text { Dependente }\end{array}$ & $\begin{array}{c}\text { Desigualdade de } \\
\text { Renda }\end{array}$ & Índice de Gini & $\begin{array}{c}\text { Intervalo de } 0 \text { à } \\
1\end{array}$ & - & Censo IBGE \\
\hline \multirow{3}{*}{$\begin{array}{c}\text { Variáveis } \\
\text { Explicativas } \\
\text { (X) }\end{array}$} & $\begin{array}{l}\text { Crescimento } \\
\text { (PIB } p c)\end{array}$ & $\begin{array}{l}\text { PIB per capita } \\
\text { Municipal }\end{array}$ & $\begin{array}{l}\mathrm{RS} \text { de } 1991 \\
/ 2000 / 2010\end{array}$ & Positivo & Censo IBGE \\
\hline & $\begin{array}{l}\text { Crescimento } \\
\left(\mathrm{PIB} p c^{2}\right)\end{array}$ & $\begin{array}{l}\text { PIB per capita } \\
\text { Municipal }^{2}\end{array}$ & $\begin{array}{c}\mathrm{R} \$ \text { de } 1991 \\
/ 2000 / 2010^{2}\end{array}$ & Negativo & $\begin{array}{l}\text { Calculada } \\
\text { de } X\end{array}$ \\
\hline & $\begin{array}{l}\text { Crescimento } \\
\quad\left(\mathrm{PIB} p c^{3}\right)\end{array}$ & $\begin{array}{l}\text { PIB per capita } \\
\text { Municipal }^{3}\end{array}$ & $\begin{array}{l}\mathrm{RS} \text { de } 1991 \\
/ 2000 / 2010^{3}\end{array}$ & Positivo & $\begin{array}{c}\text { Calculada } \\
\text { de } X\end{array}$ \\
\hline
\end{tabular}

Fonte: Oliveira et al. (2011) - Adaptado.

Baseada na função estimada, ressalta-se que os coeficientes $\beta_{i}$ da equação acima determina a relação entre as variáveis (proxies), o crescimento econômico (renda per capita), a qual pode apresentar vários possíveis resultados, dependendo do desempenho de cada economia (DE BRUYN; VAN DEN BERGH; OPSCHOOR, 1998). Os possíveis resultados estão descritos no quadro a seguir: 
Desenvolvimento Econômico na Região da Riba a partir da Relação Crescimento-Desigualdade sob Hipótese(s) da(s) Curva(s) de Kuznets

Quadro 2. Resultados da função estimada na regressão da Curva de Kuznets

\begin{tabular}{|c|c|}
\hline$\hat{\beta}_{1}=\hat{\beta}_{2}=\hat{\beta}_{3}=0$ & Nenhuma relação existente entre renda per capita e desigualdade de renda. \\
\hline$\hat{\beta}_{1}>0$ e $\hat{\beta}_{2}=\hat{\beta}_{3}=0$ & $\begin{array}{l}\text { A relação entre renda per capita e desigualdade de renda é monotonicamente positiva } \\
\text { e linear, onde um aumento da primeira leva a um aumento constante da segunda. }\end{array}$ \\
\hline$\hat{\beta}_{1}<0$ e $\hat{\beta}_{2}=\hat{\beta}_{3}=0$ & $\begin{array}{l}\text { A relação entre renda per capita e desigualdade de renda é monotonicamente } \\
\text { negativa e linear. }\end{array}$ \\
\hline$\hat{\beta_{1}>0}$ e $\hat{\beta}_{2}<0$ e $\beta_{3}=0$ & $\begin{array}{l}\text { A relação entre renda per capita e desigualdade de renda pode ser representada pela } \\
\text { CKT - função quadrática com concavidade para baixo (na forma de "U invertido"). }\end{array}$ \\
\hline$\hat{\beta_{1}<0}$ e $\hat{\beta}_{2}>0$ e $\beta_{3}=0$ & $\begin{array}{l}\text { Oposição ao formato de "U invertido". A relação entre renda per capita e desigualdade } \\
\text { de renda traduz-se através de uma função quadrática com concavidade para cima, } \\
\text { não podendo ser representada pela CKT. }\end{array}$ \\
\hline$\hat{\beta_{1}>0}$ e $\hat{\beta}_{2}<0$ e $\beta_{3}>0$ & $\begin{array}{l}\text { A relação entre renda per capita e desigualdade de renda passa a assumir uma relação } \\
\text { na forma de "N", revelada numa função polinomial cúbica. Nesse caso, a CKT } \\
\text { representaria apenas um estágio daquela relação, anterior ao "recasamento" a partir } \\
\text { do segundo ponto de inflexão da curva. }\end{array}$ \\
\hline$\hat{\beta}_{1}<0$ e $\hat{\beta}_{2}>0$ e $\hat{\beta}_{3}<0$ & $\begin{array}{l}\text { Oposição ao formato de "N". Revelada numa função polinomial cúbica de formato "N } \\
\text { invertido". }\end{array}$ \\
\hline
\end{tabular}

Fonte: CARVALH0 (2008); CIRIACI e PALMA, 2009); DINDA (2004); LUCENA (2005) - Adaptado.

Na aplicação da curva de Kuznets a especificação tradicional do modelo inclui a variável renda per capita e seu termo quadrático (BARBIER; BURGESS, 2002; SALVAT0 et al., 2006; TAQUES; MAZZUTI, 2009; SANTOS; CUNHA; GADELHA, 2017), alguns trabalhos também incluíram a forma cúbica na análise (TABOSA; FILHO; GOMIDE, 2016; BARROS; HENRIQUES; MENDONÇA, 2000; LINHARES et al., 2012; RODRIGUES, 2014). Cabe enfatizar que, a partir de uma função cúbica Tribble (1996) descreveu um outro formato da curva de Kuznets descrito como "S".

A diferenciação nas formas funcionais do PIB per capita se dá de acordo com o intuito do tipo de curva - quadrática ou cúbica, a ser testada no modelo. 0 PIB per capita ao quadrado é incluído para testar a hipótese de que o a desigualdade de renda cresce a taxas decrescentes para níveis mais baixos de renda, e a partir de certo ponto, a elevação do nível de renda reduza desigualdade. 0 termo cúbico é incluído para testar se a relação segue uma forma de "N", ou seja, após a redução, a desigualdade voltaria a aumentar com o nível de renda (OLIVEIRA et al., 2011).

Dependendo dos sinais dos coeficientes estimados na equação da regressão, a função estimada assume formatos distintos, ilustrando tipos diferentes das curvas que denotam a relação entre crescimento econômico (renda per capita) e desigualdade de renda (Gini) (DE BRUYN; VAN DEN BERGH; OPSCHOOR, 1998).

\section{RESULTADOS E DISCUSSÃO}


Os comportamentos do indicador de desigualdade (índice de Gini) e do crescimento econômico (PIB per capita) para os municípios da RIBA mostraram-se ascendentes ao longo de todo o período analisado, 2000-2010.

De acordo com 0 gráfico de dispersão (figura 1), nota-se que houve uma aumento mais acentuado no índice de Gini comparativamente ao PIB per capita dos anos de 1990 até 2000. A partir de então, o PIB per capita aumentou duramente.

A medida do grau de relacionamento entre duas variáveis é chamada de coeficiente de correlação. Esta apresentou para as proxies índice de Gini e renda per capita um valor de 0,65942743, com p-valor = 0,0000 para a $H_{0}$ de não correlação.

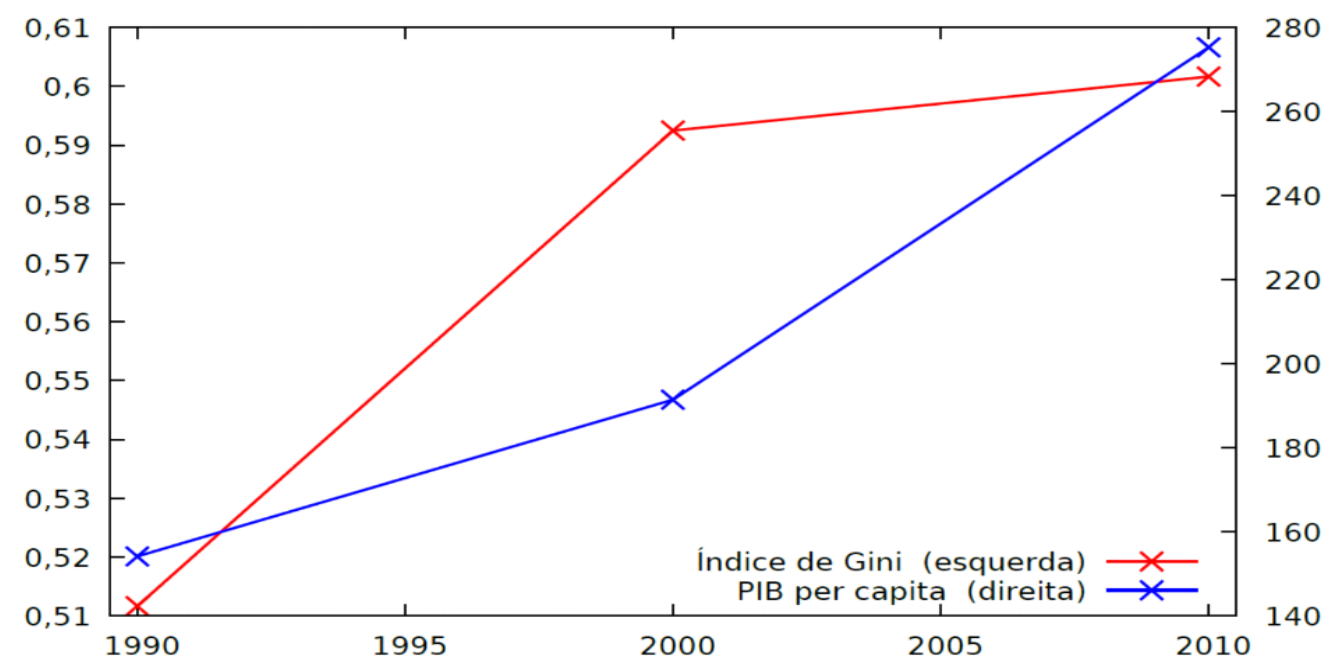

Figura 1. Dispersão das Variáveis Índice de Gini e PIB per capita em Painel.

Fonte: Elaboração dos autores com uso do Gretl.

0 modelo estimado analisa a(s) hipótese(s) de uma curva de Kuznets em forma de "U invertido" e "N", utilizando as variáveis renda per capita, na qual seus termos ao quadrado e cúbico foram considerados. A partir dos resultados dos coeficientes relacionados à renda per capita verificou-se que todos apresentaram os sinais esperados e mostraram-se estatisticamente significantes, ao nível de 5\% e 10\%, podendo aceitar a hipótese de uma curva com formato de "N" para a RIBA (relações linear, quadrática e cúbica das rendas per capitas) com a desigualdade, resultados da estimação do modelo de regressão com dados em painel (predominância pela escolha do modelo pooled - teste Chow, na decisão da escolha; teste Hausman que indicou o efeito aleatório preferível ao de efeito fixo e, o teste de Breusch Pagan selecionou o modelo pooled ao de efeito aleatório) relacionando o crescimento econômico com a desigualdade de renda (equação 5) conforme dispostos na Tabela 1 e equação (5):

Tabela 1. Resultados do Modelo (MMQ0) e testes de significância das variáveis 
Desenvolvimento Econômico na Região da Riba a partir da Relação Crescimento-Desigualdade sob Hipótese(s) da(s) Curva(s) de Kuznets

\begin{tabular}{|c|c|c|c|c|}
\hline Parâmetros & Constante & Renda_pc & Renda_p $c^{2}$ & Renda_pc ${ }^{3}$ \\
\hline $\begin{array}{c}\text { Coeficientes } \\
\text { (Erro Padrão) } \\
\text { Razão-t } \\
\text { p-valor }\end{array}$ & $\begin{array}{c}0,250418 \\
(0,105977) \\
2,363 \\
0,0244^{* *}\end{array}$ & 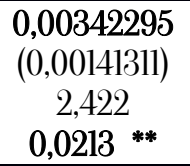 & $\begin{array}{c}-1,11950 \mathrm{e}-05 \\
(5,60611 \mathrm{e}-06) \\
-1,997^{*} \\
\mathbf{0 , 0 5 4 4}^{*}\end{array}$ & $\begin{array}{c}1,22821 \mathrm{e}-08 \\
(6,61853 \mathrm{e}-09) \\
1,856 \\
0,0727^{*}\end{array}$ \\
\hline
\end{tabular}

Fonte: Resultados do Gretl com base nos dados dos Censos Censitários.

Coeficientes das variáveis explicativas significantes à *0,10p; ${ }^{* *} 0,05 p \mathrm{e}{ }^{* * *} 0,01 p$.

A equação estimada é:

$\operatorname{lnGini}=0,250418+0,00342295 \operatorname{lnRendapc}_{i t}-1,11950 \mathrm{e}-05 \operatorname{lnRendapc_{it}^{2}}+$ $1,22821 \mathrm{e}-08 \ln R e n d a p c_{i t}^{3}+\varepsilon i t$

Em caráter generalizado, a renda estaria atrelada ao processo de desigualdade na RIBA, de acordo com a hipótese da curva em formato de "N". Assim, em estágios mais avançados de renda per capita, a desigualdade de renda voltaria a aumentar em função do crescimento econômico, assim, a redução da desigualdade não se manteria, de acordo com a evidência empírica encontrada no trabalho pioneiro de List e Gallet (1999) sobre este formato da curva de Kuznets. De acordo com o modelo em sua forma polinomial cúbica, a Curva de Kuznets está disposta na Figura 2.

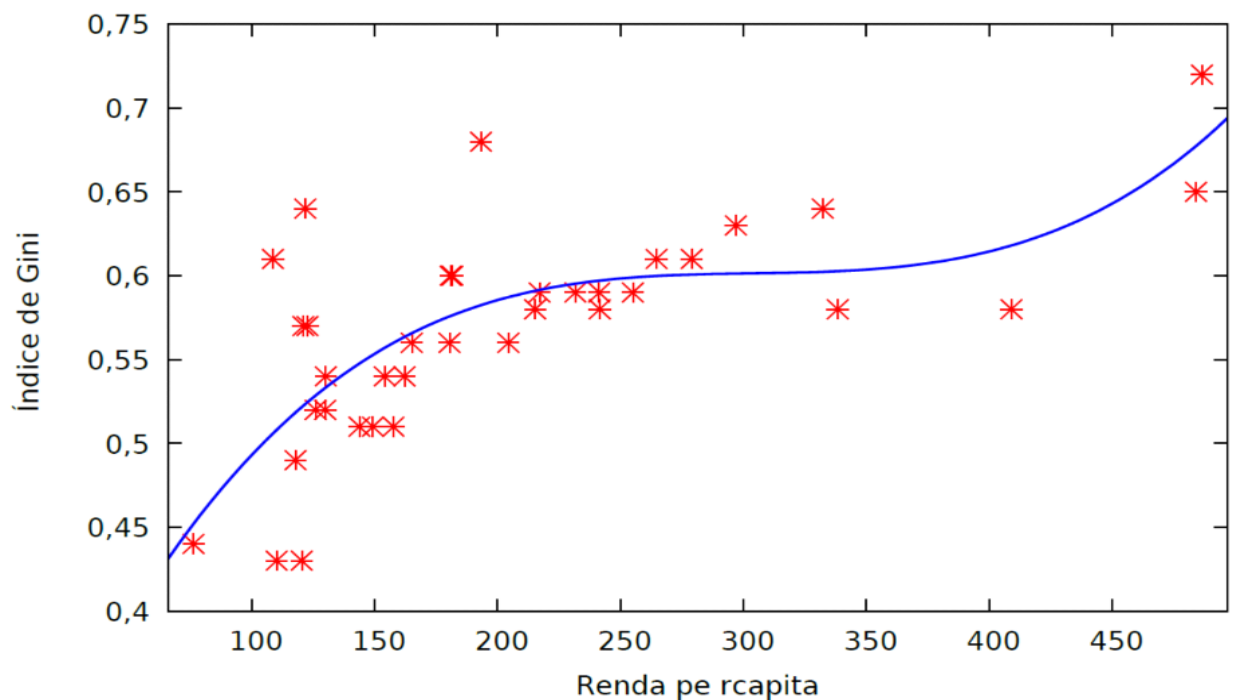

Figura 2. Dispersão das Variáveis em Painel - Estimação da CKT Fonte: Elaboração dos autores com uso do Gretl.

A figura acima, demonstra que a relação entre o PIB per capita e o índice de desigualdade de renda (Gini) assumiu uma relação no formato parecido à forma de “N”, revelada pela função polinomial cúbica da curva de Kuznets. Logo, os resultados do modelo acima evidenciaram uma possível junção (casamento) de duas curvas de formatos "U invertido" e "U” (apesar de não constar explicitamente, a estimação da regressão sob a forma quadrática, teve seus parâmetros 
significativos ao nível de 1\%) podendo, dessa forma, caracterizar dois estágios diferentes de progresso econômico vivenciado na região, com impactos distintos sobre o nível de desigualdade de renda no período analisado, sendo estes: i) crescente-decrescente (primeiro estágio) e ii) decrescente-crescente (segundo estágio). Ou seja, o aumento contínuo do nível de renda proporcionou uma melhora relativa e temporária no nível de desigualdade de renda (na faixa de renda média que varia entre $\mathrm{R} \$ 200,00$ e $\mathrm{R} \$ 400,00)$ voltando a desigualdade a subir de patamar com a contínua elevação da renda. Isto, levando também em consideração os sinais e os valores dos parâmetros da equação (5).

A existência da relação cúbica não necessariamente inválida a proposta inicial de Kuznets, pois como argumentado no referencial teórico da hipótese do U invertido, numa economia com diversos setores, é possível que a relação crescimento-desigualdade se reverta em função do surgimento de um setor mais novo e atrativo, de forma a elevar a desigualdade novamente (TABOSA, FILHO e GOMIDE, 2016).

0 surgimento dos setores de alta tecnologia, intensivos em capital humano especializado, a expansão do setor de serviços e o enfraquecimento do Estado, poderiam ser responsáveis pela finalização da fase decrescente/contante e 0 início de uma nova fase de crescimento na desigualdade. No período analisado a evidência da hipótese do U invertido da curva de Kuznets se fez presente no primeiro estágio do progresso econômico da região, corroborando com Tabosa, Filho e Gomide (2016).

Além da estimação da Curva de Kuznets, também foram realizados os testes de violação dos pressupostos básicos do modelo da regressão: normalidade dos resíduos, não linearidade (quadrados e logaritmo), especificidade - pelo critério Ramsey/Reset (quadrado), heterocedasticidade - pelo critério de White e autocorrelação - pelo critério de Durbin-Watson, conforme descritos na tabela abaixo:

Tabela 2. Resultados dos Testes de Violação dos Pressupostos do MMQ0

\begin{tabular}{c|c|c|c}
\hline TESTE & $\mathrm{p}$-valor & TESTE & $\mathrm{p}$-valor \\
\hline Especificidade & 0,438358 & Normalidade & 0,120518 \\
\hline Não-Linearidade (quadrados) & 0,469504 & Heteroscedasticidade & 0,307273 \\
\hline Não-Linearidade (Logaritmo) & 0,769033 & Autocorrelação DB & 0,760263 \\
\hline
\end{tabular}

Fonte: Resultados do Modelo com base nos dados Secundários.

Os resultados calculados para os testes revelam que as especificações utilizadas são adequadas, podendo tecer inferências, a partir da obtenção das estimativas de beta relativas aos valores dos parâmetros populacionais. 
Desenvolvimento Econômico na Região da Riba a partir da Relação Crescimento-Desigualdade sob Hipótese(s) da(s) Curva(s) de Kuznets

\section{CONCLUSÃ0}

Considerando que Kuznets (1955) buscou analisar e compreender o comportamento da desigualdade a medida que crescimento econômico evolui positivamente, postulado na existência de uma relação linear entre crescimento econômico e desigualdade de renda na forma de "U invertido", indicando que na dinâmica da desigualdade ao longo do tempo se eleva, estabilizando em seguida e, se reduz posteriormente à medida que a economia continua a crescer.

Retomando os resultados da revisão, parece longe de se avistar um consenso no campo conforme das discussões teóricas quanto nas evidências empíricas, sobre a existência de uma padronização da relação entre crescimento e desigualdade. Com respaldado no fato dos estudos se basearem em amostras, variáveis e metodologias diferentes, o que levam os resultados encontrados dificilmente convergirem para uma conclusão definitivamente comum.

A contribuição deste trabalho para a literatura foi testar a validade CKT em recorte municipal (dados cross sections) associada a uma escala de temporal decenal de trinta anos (serial temp), com dados sob a estrutura de dados em painel intercalado. No modelo estimado todos os coeficientes dos termos ligados à renda per capita apresentaram o sinal para uma curva com a forma de "N", onde os coeficientes mostraram-se estatisticamente significante ao nível de $5 \%$ e 10\% para as variáveis que representam a renda e suas relações linear, quadrática e cúbica respectivamente com a desigualdade de renda, evidenciando, em caráter generalizado, que a renda estaria atrelada ao processo de desigualdade na RIBA, em dois momentos antagônicos da relação entre crescimento econômico e desigualdade de renda.

Os resultados do modelo, de forma atenuada, evidenciaram uma possível junção (casamento) de duas curvas de formatos “U invertido" e " $U$ ”, caracterizando, de fato dois estágios (momentos) diferentes de progresso macroeconômico vivenciado na região, com impactos distintos sobre o nível de desigualdade de renda no período analisado, apresentando-se de forma crescente-decrescente e decrescente-crescente no primeiro e segundo estágio, respectivamente, o que, por sua vez não necessariamente invalidou a proposta inicial de Kuznets baseado na hipótese de Kuznets do U invertido, pois em uma economia com diversos setores, é possível que a relação crescimento-desigualdade se reverta em função do surgimento de um setor mais novo e atrativo, de forma a elevar a desigualdade novamente.

Por fim, na RIBA no período analisado (1990-2010) há evidencia da hipótese do U invertido presente no primeiro estágio do progresso econômico na região, corroborando o pressuposto 
teórico de Kuznets, com base nos estudos pioneiro de List e Gallet (1999), atualmente constatado no estudo de Tabosa, Filho e Gomide (2016), muito embora, em níveis agregados, a influência da evolução crescente da renda per capita possa ser interpretado, também, como apaziguador na evolução crescente da desigualdade de renda, atuando assim, o crescimento econômico como pacificador momentâneo junto à desigualdade de renda na região.

\section{REFERÊNCIAS BIBLIOGRÁFICAS}

ARRAES, R. A; DINIZ, M. B.; DINIZ, M. J. T. Curva ambiental de Kuznets e desenvolvimento econômico sustentável. Rev. de Econ. e Sociologia Rural, Rio de Janeiro, v. 44, n. 03, p. 525-547, jul./set. 2006.

BARBIER, E. B; BURGESS, J. C. The Economics of Tropical Deforestation. Journal of Economic Surveys, v. 15, n. 3, p. 413-432. 2002.

BARBOSA, William; OLIVEIRA, Edivo de Almeida; SILVA, João Victor Souza da; FREITAS, Clailton Ataídes de. Uma análise da curva de Kuznets para os municípios brasileiros (1991, 2000, 2010). Economia \& Região, Londrina, Paraná, v. 5, n.l, p. 65-83, jan./jun. 2017.

BARROS, R. P. de; HENRIQUES, R.; MENDONÇA, R. Desigualdade e Pobreza no Brasil: retrato de uma estabilidade inaceitável. Revista Brasileira de Ciências Sociais, São Paulo, v.15 n. 42, fev. 2000.

CARVAlHO, T. S. A Hipótese da Curva de Kuznets Ambiental Global e o Protocolo de Quioto. 2008. 130 f. Dissertação (Mestrado). Faculdade de Economia e Administração, Universidade Federal de Juiz de Fora, Juiz de Fora. 2008.

CIRIACI, D.; PALMA, D. Geography, enviromental efficiency and economic growth: how to uncover localized externalities through spatial econometric modeling. SEA, III World Conference, Barcelona, 2009. Disponivel em:< http://www.ub.edu/sea2009.com/Papers/113.pdf>. Acesso em: 14 ago. 2018.

DE BRUYN, S. M.; VAN DEN BERGH, J. C. J. M.; OPSCHOOR, J. B. Economic growth and emissions: reconsidering the empirical basis of environmental Kuznets curves. Ecological Economics, v. 25, p. 161-175, 1998.

DEUTSCH, J.; SILBER, J. The kuznets curve and the impact of various income sources on the link between inequality and development. Working Paper Bar-Ilan University, Israel, 2000.

DINDA, S. Environmental Kuznets Curve Hypothesis: A Survey. Ecological Economics. v. 49, p. 431-455, 2004.

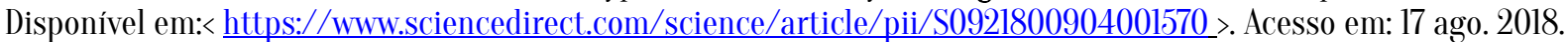

FAPESPA, 2017. Mapa da Região de Integração do Baixo Amazonas (RIBA) disponível em < http://www.fapespa.pa.gov.br/sistemas/radar2017/mapas/01_territorio/regiao_de_integracao_baixo_amazonas.pn g >. Acesso em: 19 mar. 2019.

GUJARATI; D. N. Econometria Básica. 4 ed. Rio de Janeiro: Elsevier- Campus, 2006.

GUJARATI, D. N.; PORTER, D. C. Econometria Básica. 5 ed. Porto Alegre: RS, 2011.

JONES, C. Introdução à teoria do crescimento econômico. Tradução de Maria Jose Cyhlar Monteiro. Rio de Janeiro: Elsevier, 2000.

KUZNETS, S. Economic Growth and Income Inequality. American Economic Review, Pittsburgh, v. 45, n.l, 1955. p. 128. 
Desenvolvimento Econômico na Região da Riba a partir da Relação Crescimento-Desigualdade sob Hipótese(s) da(s) Curva(s) de Kuznets

LINHARES, F.; FERREIRA, R. T.; IRFFI, G. D.; MACEDO, C. M. B. A hipótese de Kuznets e mudanças na relação entre desigualdade e crescimento de renda no Brasil. Pesquisa e planejamento econômico, Rio de Janeiro, v. 42, n. 3, p. 403-432, dez. 2012.

LIST, J. A.; GALLET, C. A. The Kuznets curve: what happens after the inverted-U. Review of Development Economics, v. 3, n. 2, p. 200-206, fev/1999.

LUCENA, A. F. $P$ de. Estimativa de uma Curva de Kuznets Ambiental aplicada ao uso de energia e suas implicações para as emisões de carbono no Brasil. Dissertação (Mestrado em Planejamento Energético) - Faculdade de Engenharia da UFRJ, Rio de Janeiro, 2005.

OLIVEIRA, R. C. de; ALMEIDA, E.; FREGUGLIA, R. da S.; BARRETO, R. C. S. Desmatamento e Crescimento Econômico no Brasil: uma análise da Curva de Kuznets Ambiental para a Amazônia Legal. Rev. de Economia e Sociologia Rural. Piracicaba - SP, v. 49, n.3, p. 709-740, jul/set, 2011.

RODRIGUES, R. T. RELAÇÃ0 ENTRE DESIGUALDADE E RENDA NOS MUNICÍPIOS BRASILEIROS: 0 que mudou na década 2000-2010? Dissertação apresentada ao Programa de Pós-Graduação em Ciências Contábeis da Fundação Instituto Capixaba de Pesquisas em Contabilidade, Economia e Finanças (FUCAPE), Espirito Santo, 2014.

SACHS, I. Desenvolvimento: Includente, Sustentável, Sustentado. Rio de Janeiro: Garamond, 2008.

SALVATO, M. A.; ALVARENGA, P. S.; FRANÇA, C. S.; JUNIOR, A. F. de A. Crescimento e desigualdade: evidências da Curva de Kuznets para os municípios de Minas Gerais - 1991/2000. Working Paper n. 33 Ibmec, Minas Gerais, 2006.

SANTOS, W. 0.; MOURA, F. R. de; SILVA, A. R. S.; MATOS, D. L.; FARIAS, T. A.. A teoria do U invertido: um teste da hipótese de Kuznets para a relação entre crescimento econômico e desigualdade de renda no Brasil (1976-2007). Revista de Economia, Paraná, v. 37, n. 2, p. 7-28, maio/ago. 2011.

SANTOS, M. P. dos; CUNHA, M. S. da; GADELHA, S. R. de B. Distribuição de renda e desenvolvimento econômico: análise da hipótese de Kuznets para os estados brasileiros no período 1992-2010. Revista Brasileira de Estudos Regionais e Urbanos, v. 11, n. 2, p. 251-271, 2017.

SILVA, M. 0. da S. e. Pobreza, desigualdade e políticas públicas: caracterizando e problematizando a realidade brasileira. Revista Katálysis. Florianópolis, v. 13 n. 2 p. 155-163 jul./dez. 2010.

SILVA, J. L. M. da; OTTONELLI, J.; MARIN, S. R. Elasticidade da pobreza nos domicílios nordestinos: 0 impacto da renda e desigualdade da renda (2001 a 2009). Economia e Desenvolvimento, Recife (PE), v. 12, n. 2, 2013.

SOUZA, N. de J. Desenvolvimento econômico. 3 Edição. São Paulo: Atlas,1997.

TABOSA, F. J. S.; FILHO, J. do A.; GOMIDE, U. G. Revista do Desenvolvimento Regional (REDES), Santa Cruz do Sul, v. 21, n. 02 , p. 245 - 266, mai./ago. 2016.

TAQUES, F. H.; MAZZUTTI, C. C. de T. P. da C. Curva de Kuznets: Mensuração do Impacto do Crescimento Econômico sobre a Desigualdade de renda para os Estados Brasileiros (1995-2005). In: XIV Encontro Regional de Economia do Nordeste, 2009, Fortaleza, 2009.

TAQUES, F. H.. Qual a relação entre desigualdade de renda e nível de renda per capita? testando a hipótese de Kuznets para as unidades federativas brasileiras. Revista Planejamento e Políticas Públicas, Brasilia, n. 35, p. 161-186, 2010.

TRIBBLE, R. The Kuznets-Lewis Process within the Context of Race and Class in the U. S. Economy. International Advances in Economic Research, v. 2, p. 151-64. 1996.

VEIGA, J. E. da. Desenvolvimento sustentável: o desafio do século XXI. Rio de Janeiro: Garamond, 2010. 